\title{
Contexto y exigencias a las ciencias sociales, para afrontar los problemas ambientales
}

\author{
María Luisa Eschenhagen \\ Universidad Pontificia Bolivariana, Medellín, Colombia. \\ Email: mariesche22@yahoo.com.mx
}

\begin{abstract}
Resumen: El propósito de este trabajo es mostrar, primero, cómo se formaron las ciencias sociales, con qué características y presupuestos, y comprender sus dificultades para entender y abordar los problemas ambientales. En un segundo momento se presentarán unas reflexiones en torno al conocimiento y su relación con el ambiente, así como la importancia de superar la escisión entre las llamadas ciencias duras y las sociales/humanas, y la necesidad de aportes como los de las ciencias de la complejidad, de los estudios culturales, de modernidad/ colonialidad, y del pensamiento ambiental, como desafíos y posibilidades para abordar el problema ambiental de manera integral. Este conjunto de aproximaciones representa un reto grande para las ciencias sociales, pero sin enfrentarlo será imposible abordar los problemas ambientales de manera adecuada.

Palabras clave: Ciencias sociales, conocimiento, problemas ambientales, modernidad/colonialidad, estudios culturales, pensamiento ambiental, paradigmas emergentes
\end{abstract}

\section{Context and requirements to social sciences, to confront environmental problems}

\begin{abstract}
The purpose of this work is to show how social sciences emerged, with which characteristics and assumptions, and, therefore, which difficulties they have to understand and to deal with environmental problems. In a second moment, there will be presented some analysis about knowledge and its relationship with the environment, as well as the importance to overcome the division between the so-called hard sciences and the social and human sciences, and the need of contributions of the sciences of complexity, cultural studies, modernity/coloniality studies and environmental thought, both as challenges and possibilities to treat environmental problems in an integral way. These combined approximations represent a great task for the social sciences, but, without them, it will be impossible to deal with the environmental problems in an adequate way.

Key words: Social sciences, knowledge, environmental problems, modernity/coloniality, cultural studies, environmental thought, emergent paradigms
\end{abstract}

\section{Contexto e requisitos as ciências sociais para resolver os problemas ambientais}

Resumo: O objetivo deste trabalho é mostrar, em primeiro lugar, como se 
formaram as ciências sociais, com que características e orçamentos, e compreender as suas dificuldades na compreensão e resolução dos problemas ambientais. Na segunda etapa irá apresentar algumas reflexões sobre o conhecimento e sua relação com o ambiente, bem como a importância de superar a divisão entre as chamadas ciências duras e as ciências sociais / humanas, ea necessidade de contribuições como as ciências da complexidade, dos estudos culturais da modernidade / colonialidade e do pensamento ambiental, como desafios e possibilidades de resolver os problemas ambientais de forma holística. Este conjunto de aproximações é um grande desafio para as ciências sociais, mas sim enfrentá-lo será impossível responder a questões ambientais de forma adequada.

Palavras-chave: ciências sociais, conhecimento, questões ambientais, modernidade / colonialidade, estudos culturais, pensamento ambiental, paradigmas emergentes

\section{Preámbulo}

El mundo de hoy parece girar cada vez más vertiginosamente. Los problemas con los que tienen que lidiar los gobiernos, las comunidades y los individuos son cada vez más complejos, entendiendo acá por complejidad no el hecho de que los problemas sean complicados, sino la imposibilidad de analizarlos y comprenderlos de manera simple, lineal y fragmentada, pues tienen un sinnúmero de causas y efectos interrelacionados. Existen, por un lado, problemas que no son nuevos y que siguen girando en torno a temas como desintegración de redes sociales, violencia, guerras, injusticias económicas y sociales, ideologías y democracias inciertas, autoritarismos, pero ahora aparecen, con más impacto y visibilidad, problemas ambientales por doquier.

En la sociedad moderna se tiene la costumbre de explicar las situaciones socioeconómicas y buscarles soluciones a los problemas a través de las ciencias sociales, soluciones que luego son aplicadas en las comunidades y sociedades por quienes toman las decisiones, los planificadores, a través de programas y proyectos. Se supone, como bien lo señala López Segrera, que "el papel esencial de los científicos sociales consiste en iluminar a los tomadores de decisiones con respecto a las opciones posibles ante las alternativas históricas” (2000: 11).

Ahora bien, al contemplar un panorama en el que los problemas se agudizan, pareciera que las ciencias sociales no estuvieran proporcionando ayuda y apoyo suficientes y/o satisfactorios. Por lo tanto, se plantean preguntas como las siguientes: ¿con qué marcos y teorías explicativas tratan de responder las ciencias sociales a la compleja realidad socioeconómicaambiental?, ¿qué tan flexibles son los supuestos y racionalidades de las ciencias sociales para comprender situaciones complejas?, ¿por qué, por ejemplo, frente a problemas ambientales, resultan insuficientes los enfoques tradicionales?, ¿existen otros caminos posibles para conocer el mundo y, por ende, otras soluciones que no pasen a través de las ciencias mo- 
dernas? Son éstas las preguntas que van a guiar este artículo, con el propósito de abrir la discusión acerca de la importancia de nuevos enfoques en las ciencias sociales (como el saber ambiental y la complejidad) para abordar los problemas socioambientales. El propósito central será mostrar algunos aspectos de cómo se formaron las ciencias sociales, con qué características y por ende con qué dificultades se encontraron para comprender los problemas actuales como los ambientales. Finalmente, se señalarán algunas propuestas que se vienen forjando en diferentes espacios, para caminar por senderos nuevos con perspectivas y posibilidades diferentes. De ahí que el propósito de este texto sea incentivar y aportar al objetivo de las ciencias sociales tradicionales, y reconocer el encierro (para salir de él) en que en muchos casos aún se encuentran.

\section{Breve historia del surgimiento y contextualización de las ciencias sociales}

Para poder entender mejor las ciencias sociales, resulta necesario ubicar su surgimiento y contexto, tanto histórico como geográfico, ya que representan una forma muy particular de aproximarse y de conocer el mundo.

Con la conquista y colonización del continente americano comienza una nueva etapa en la historia de la humanidad, y más específicamente para los continentes americano y europeo. Con este evento no se da solamente un cambio en la percepción y dominio de un espacio físico ampliado, sino también una mudanza en la cosmovisión, específicamente en y desde la visión europea. Es en ese momento cuando ocurre una transición de una visión geocentrista/teológica del mundo a una heliocentrista racional/ mecanicista.

La visión racional y mecanicista nace en Europa Central, es decir, tiene un lugar de "nacimiento" y un contexto histórico social específicos. La conquista espacial de América va acompañada por grandes cambios tanto sociales como de pensamiento en la propia Europa; estos cambios se dan de forma lenta, a manera de procesos, y no exactamente de manera pacífica: son múltiples las guerras y revoluciones para cambiar y/o imponer las ideas. Este nuevo contexto requiere del surgimiento de pensadores como Copérnico y Galileo para comenzar a dominar la navegación; requiere de filósofos como Descartes para salir del mundo teológico, que ya se había quedado corto para dar respuestas satisfactorias a las nuevas necesidades (se requería ver el mundo ahora con un conocimiento que pretendía ser objetivo, universal y verdadero); y de físicos como Newton, para comenzar a fortalecer la industria naciente. Pero también el creciente mercantilismo requería cada vez más de especialistas -con sus conocimientos específicos- y/o de empleados públicos para organizar las nuevas relaciones socioeconómicas. Europa se rebelaba en contra de la monarquía, cuya jerarquía y organización sociopolítica ya no eran capaces de satisfacer las exigencias emergentes y diversas del mundo mercantilista. Por lo tanto, el 
continente entero eliminó la monarquía en arduas guerras y se inventó, en un largo proceso, una nueva organización socioeconómica, la del Estadonación democrático, y se creó un sistema económico, el capitalismo. Con este nuevo panorama comenzaron a surgir necesidades de conocimientos relacionados con estadísticas, jurisprudencia, administración y economía política, para organizar el manejo y funcionamiento de los Estados-nación.

Es en este ambiente donde se comienzan a consolidar las universidades y con ellas se va fortaleciendo la ciencia moderna, como método específico para conocer y dominar el mundo, método que pretende posibilitar un conocimiento que sea objetivo, verdadero y universalmente válido. Entre esas ciencias modernas, la física marca la pauta de qué es y cómo debe ser una ciencia. Por lo tanto, si existen leyes físicas que rigen la materia y permiten predecirla y controlarla, de igual forma se debe comenzar a observar la naturaleza y la sociedad, suponiendo que éstas deben funcionar del mismo modo, es decir, con leyes y de manera mecanicista (Comte 1996). Es así como se conciben entonces las sociedades, regidas por normas y leyes que pueden ser calculadas, planificadas, dominadas y previsibles. A su vez la naturaleza es vista como un ente inerte, uniforme y homogéneo, que debe ser descuartizado, analizado y planificado para ser usado y explotado (Bacon 1984). A través de la ciencia positiva, lo que se pretendía básicamente era liberarse de la teología, la metafísica u otras maneras de “conocer” la realidad, y, ahora sí, ser capaz de descubrir la "realidad objetiva”. El mundo se convierte así en un objeto, en una cosa distante, independiente del ser humano; de ahí que se hable del “desencantamiento" del mundo.

Con esta forma de conocer se termina de cimentar la escisión entre cuerpo y mente, entre mundo y razón, entre cultura y naturaleza. Esta escisión tiene sus raíces, según el marco histórico específico del que se parta, en los sumerios, como bien lo demuestra Elmusa (2005) en su análisis de la epopeya de Gilgamesch; o en los griegos, especialmente cuando se mira su historia desde Platón hasta llegar a Pablo de Tarso y el naciente cristianismo; Pablo es quien definitivamente realiza la separación entre Dios, hombre y naturaleza, como lo demuestra Ángel Maya (2002). Al realizar esta ruptura entre cuerpo y mente, el cuerpo y el mundo se vacían de sentido, y la razón y la mente son puestas en un pedestal incuestionable (ver también Castro-Gómez 2005). Con esta concepción, ya sólo hay que dar un pequeño paso para que se genere y se desarrolle una postura puramente instrumental frente al cuerpo y al mundo. El mundo fue así desencantado y pudo ser dominado, explotado y planificado sin mayores escrúpulos. Ya no importaron las preguntas sobre por qué y para qué, sino aquellas que se interesaban por el qué y el cómo. La filosofía quedó entonces relegada y desvirtuada (ver la historia de esta escisión en Ángel Maya 2000 y 2002).

Cuando llega el siglo XIX, y tras fomentar el conocimiento “objetivo”, se comienzan a vislumbrar las ciencias sociales (por ejemplo la economía) cuando ya el capitalismo y la vida liberal se habían instaurado en Europa como formas “naturales” de vivir. Aquí no se puede olvidar, como 
bien lo señala Lander (2000), que estas transformaciones en Europa fueron "extraordinarias y traumáticas", ya que implicaron rupturas profundas en las formas de vida y de producción de los campesinos, la expulsión de muchos de sus tierras hacia las grandes urbes, y un cambio drástico de vida al introducir la disciplina del trabajo fabril. Estos cambios de ningún modo se dieron pacíficamente ni de manera natural. Lo que se dio fue más bien una imposición de un régimen de disciplina y una normatización para poder crear al homo economicus. Así, las ciencias sociales nacen ya dentro de la hegemonía del proyecto liberal y ayudan más bien a construirlo y consolidarlo; es decir, las reflexiones y teorías nacen y giran ya dentro del modelo liberal mismo y por ende tienen poca capacidad de cuestionar este nuevo modelo civilizatorio, que era parte de su propia cuna.

Las ciencias sociales, entendidas como "un cuerpo de saber sistemático sobre las relaciones sociales humanas, que se formuló y se institucionalizó en estos dos siglos [XIX y XX]" (Wallerstein 2005: 66), se construyen alrededor de la idea de modernidad, que va tomando cada vez más forma y se expresa especialmente a través del Estado-nación. Como bien señala Wallerstein, "la ciencia social era claramente una criatura, si es que no una creación, de los Estados, y tomaba sus fronteras como contenedores sociales fundamentales" (1996: 30). Estas nuevas ciencias se dividen en ciencias nomotéticas (economía, política, antropología y sociología) y la ciencia ideográfica (historia). La filosofía queda marginada. Los ejes articuladores de esa modernidad consisten, según Lander (2000: 22), en:

1. La visión universal de la historia asociada a la idea del progreso

2. La naturalización tanto de las relaciones sociales como de la "naturaleza humana” de la sociedad liberal-capitalista

3. La naturalización u ontologización de las múltiples separaciones propias de esa sociedad

4. La necesaria superioridad de los saberes que produce esa sociedad (“ciencia”) sobre todo otro saber.

Estos ejes y supuestos tienen una serie de implicaciones. Por un lado, se construye una forma muy específica y propiamente europea de conocer el mundo, que en adelante se verá como secularizado, fragmentado, simplificado, homogeneizante, con sistemas cerrados, lineales y en equilibrio. Por otra parte, estos ejes se guían por una idea teleológica de progreso, que supone que todas las sociedades pasan por las mismas etapas evolutivas, y consideran que la etapa liberal-capitalista es la más progresista y a la que todos deberán llegar tarde o temprano (esta idea de progreso se convierte más tarde en la idea de desarrollo y se refleja en teorías sociales como la de Spencer (1905), y en teorías económicas como la de Rostov (1963)). Respecto a las separaciones múltiples (por ejemplo, cuerpo-mente, sujeto-objeto), éstas terminan por ser ontologizadas (es decir, se les adjudica un ente, una esencia propia) y se toman como "naturales" (como algo obvio, propio, esencial). En estas separaciones se destacan "lo" económico, "lo" social, "lo" cultural y "lo" natural; esto dificulta y hasta impide ver sus interrelaciones e interdependencias, así como su propio origen 
común de entendimiento/lectura desde una cultura específica, la europea occidental. Otra implicación importante de estos ejes es la consolidación de la ciencia como “el” conocimiento único y universalmente verdadero y objetivo, con lo cual se subyugan, se eliminan, se desestiman y se desprestigian otras formas de conocer y de saber (indígenas, campesinas, afros, etc.)

Esta forma científica de conocer se reduce además a subdividir, cuantificar y recombinar, y hace énfasis en el “cómo” y desplaza la pregunta sobre el "por qué”. En este contexto ya no cabe preguntar “¿esto es bueno?”, sino que solamente es considerado importante interrogarse “¿esto funciona?”. Esta aproximación no es válida exclusivamente para las llamadas ciencias duras, sino también para las ciencias sociales. Para Wallerstein (2005: 66) "los sociólogos eran físicos newtonianos en potencia”, cuyo padre fue Comte (1996). En este desplazamiento de las preguntas suceden también dos cosas: por un lado, al centrarse en la cuestión del cómo, la necesidad de lo cuantificable se hace evidente y se da de manera abstracta, con números y teorías abstractas. Esto tiene como consecuencia que el ser humano se distancie de lo tangible, de lo inmediato, así como de lo significativo, y todo (incluidos los seres humanos y el resto de seres vivos) se convierta en cosas manipulables, transformables, sustituibles. Por otro lado, se crea una valoración, un axioma, que asocia los resultados cuantitativos con lo verdadero y a su turno lo verdadero con la utilidad, particularmente con aquella industrial, lucrativa. La naturaleza (e incluso los seres humanos y las comunidades) es ya solamente valorada en relación con algo: todo debe ser Zweckrational, el mundo se convierte por lo tanto en "una colección de materia inerte que se mueve rápidamente sin fin ni significado” (Berman 2001: 45). De ahí también nace la convicción de tener la capacidad perfecta de manipular, controlar y predecir el entorno natural. Una naturaleza viva -entendida como dinámica, aleatoria, cambiante, impredecible- es un obstáculo. O, como dice Castro-Gómez: “el papel de la razón científico-técnica es precisamente acceder a los secretos más ocultos y remotos de la naturaleza con el fin de obligarla a obedecer nuestros imperativos de control” (2000: 146).

Es necesario tener presente que todos estos cambios en las formas de pensar y conocer, de vivir, de producir, no se dan de manera lineal, causal, sino más bien de manera simultánea, y que se potencian y realimentan mutuamente. Una parte importante de este proceso es la disciplinarización, tanto intelectual como profesionalizante, de esta nueva forma de concebir el mundo, que se materializa de manera muy real, aunque las interrelaciones entre las formas de conocer y lo concreto, cotidiano, no necesariamente resultan evidentes y visibles (ver también: Escobar 1996). Respecto a la disciplinarización intelectual, se ve el surgimiento de las distintas áreas de conocimiento, cada una con sus propios métodos, normas y disciplinas para asegurar su reconocimiento científico. Respecto a la disciplinarización profesionalizante, esta fragmentación del conocimiento se refleja en la aparición de las diferentes facultades de las universidades, así como en el surgimiento de profesiones específicas con tareas particulares para suplir las 
necesidades de conocimiento especializado en los diferentes ámbitos sociales (económico, jurídico, político, etc.). Pero no solamente en las universidades se da el proceso de disciplinarización, pues éste se traslada también a las escuelas. Así, cuando emerge el concepto de currículum a comienzos del siglo XX por necesidades del capitalismo, las nociones administrativas dominan en la teoría curricular poderosamente, en la organización de las actividades y el tiempo de la educación. Una cita de Cubberly, en 1918, muestra claramente la visión de educación que se tenía en aquel momento: "nuestras escuelas son, en un sentido, fábricas en las cuales la materia prima (niños) están para ser modelados y adaptados como productos” (en Giroux 1991: 32). También la cita de Bobbit, de la misma época, es muy representativa: "la función del responsable de la elaboración del plan de estudios es comparable a la de un ingeniero" (en Díaz 1991: 54). Igualmente es importante no olvidar la influencia que tenía en aquella época el modelo económico tayloriano, con sus principios de eficiencia, control y predicción. Esta situación da origen a un 'nuevo' pensamiento pedagógico que se nutre de tres vertientes conceptuales: el funcionalismo (tecnología educativa), el pragmatismo (teoría curricular) y el conductismo (evaluación) (Díaz 1991: 46). Este panorama muestra cómo la educación, y por ende buena parte de las ciencias educativas, está destinada claramente a la producción y a la industria, donde la ética ya no tiene espacio (ver también: Eschenhagen 2009).

Las personas quedan convertidas, en esta visión, en objetos, que deben ser educados y disciplinados para funcionar de manera exacta en un sistema determinado (educativo, burocrático, administrativo, fabril, etc.), en pro de la producción capitalista. Con el fenómeno de la especialización se genera también una división/separación significativa entre los llamados especialistas y la sociedad misma, división por la cual los primeros son los que saben qué es lo mejor para la sociedad (en educación, salud, vivienda, etc.), aspectos que antes, en épocas y contextos no modernos, eran decididos por las propias comunidades.

Saber qué es lo mejor para la sociedad se traduce en proyectos y posiciones políticas. Wallerstein (2001) señala, sobre todo, dos vertientes claras que se pueden reconocer, y que han entrado en debate continuamente a lo largo de los últimos cien años, aunque de manera desequilibrada, pues se ha puesto siempre a la ciencia física en una posición privilegiada. Por un lado estaban aquellos que desde la perspectiva de la física sabían que existían leyes deterministas, que, trasladadas al ámbito social, resultaban útiles para el control tecnocrático de la sociedad. Del otro lado se encontraban los que defendían lo particular, lo no determinado y lo imaginativo, que, según Wallerstein, servían para resistir al cambio tecnocrático y para reivindicar la acción humana en la esfera sociopolítica. Se sabe que la visión tecnocrática triunfó en Europa (y cada vez más en el resto del mundo) y que ha cambiado profundamente las sociedades.

Este cambio tecnocrático profundo, reflejado en los cambios tecnológicos y en el crecimiento industrial, no hubiera sido posible sin las mate- 
rias primas obtenidas de las colonias en las tierras de ultramar, que debían mantenerse dominadas para poder asegurar esta prosperidad. Pero este dominio no se dio solamente en los ámbitos militar y territorial, sino también en el campo intelectual. Sin embargo, hasta ahora, lo más común ha sido hablar del dominio y la colonización territorial que se ejercía a través de la fuerza militar y las instituciones locales impuestas, como la administración colonial (por ejemplo, las encomiendas o los ingenios) o la Iglesia, con el fin de garantizar el control y la explotación directa de las tierras. Pero se olvida, se obvia o se niega que también se dio una colonización mental/ intelectual que se mantiene hasta hoy. El problema reside en que "la mitología de la 'descolonización del mundo' oscurece las continuidades entre las jerarquías coloniales/raciales pasadas y actuales y contribuye a la invisibilidad de la 'colonialidad' de hoy en día” (Grosfoguel 2006: 29). Esto se refleja, según Grosfoguel, en las construcciones ideológicas de los últimos 50 años en los países periféricos, por ejemplo, "identidad nacional”, “desarrollo nacional” o "soberanía nacional”, que crean ilusiones de "independencia”, "progreso” y "desarrollo”. Resulta que estas construcciones teóricas tienen profundas raíces en las tradiciones filosóficas europeas y en las ciencias sociales, que, como sabemos, provenían de aquellas tierras. En realidad, estas ciencias son eurocéntricas y no recogen necesariamente los problemas concretos, endógenos de la periferia, ni posibilitan su desarrollo autóctono, autónomo o independiente. Como bien lo señala Walsh "las ciencias sociales se construyeron en las lenguas modernas de conocimiento y colonización (inglés, francés y alemán) y se ocuparon en y con la realidad de los 5 países occidentales más poderosos económicamente (Gran Bretaña, Francia, Alemania, Italia, EE.UU.)” (Walsh 2004).

Para constatar la anterior afirmación, baste con revisar todos los cánones centrales de la sociología, la economía o la política para comprobarlo. Por tal razón también se habla de una colonialidad del saber, que implica reconocer y entender que existe una hegemonía en la producción, difusión y uso del conocimiento, que fundamenta, justifica y legitima el sistema-mundo moderno capitalista (Wallerstein) y que a la vez es reproducido en los demás países ${ }^{1}$. Estas bases epistemológicas, eurocéntricas, generalmente no se cuestionan y además han sido internalizadas y asimiladas a la perfección en nuestros territorios. Además, puesto que estamos educados en esa misma visión nos resulta difícil reconocer la jaula en que nos encontramos. Adicionalmente, esos límites que imponen las bases epistemológicas tradicionales impiden ver otras opciones, racionalidades, lógicas, saberes, mundos. Indudablemente, el conocimiento está marcado geo-históricamente, geo-políticamente y geo-culturalmente, y tiene implicaciones concretas de poder y dominación, como lo demuestra el sistema-mundo moderno capitalista. Este problema se expone al plantear la geopolítica del conocimiento, como lo vienen desarrollando Mignolo y Walsh $(2004)^{2}$.

Giroux, desde sus reflexiones pedagógicas, reconoció en 1983 que el problema está en [v]isualizar el conocimiento como dominio sacerdotizado de los guerreros eruditos [lo cual] significa perder, 
como castigo, la posibilidad de cuestionar la naturaleza normativa y política del conocimiento y de los intereses sociales que ellos legitiman (Giroux 2003: 213).

Por lo tanto, el reto está en evidenciar esta profunda pérdida de cuestionamiento, reconocer la georreferencialidad del conocimiento, entender las implicaciones de poder e intereses que se juegan a través del conocimiento, así como la necesidad de repensar, replantear y abrir las ciencias sociales, con el riesgo, o más bien, con la oportunidad de que éstas desaparezcan en sus formas acostumbradas para dejar emerger otras opciones.

Pequeño interludio:

Un espacio exterior no podía, sencillamente, existir, ya que su existencia lo hubiera convertido en parte de Misraim y, con ello, en un no-espacio exterior. Lo único que existía y siempre existiría eran las catacumbas. De acuerdo con esto, toda pregunta sobre cómo se había llegado hasta ellas se consideraba un signo de ignorancia que sólo merecía una sonrisa burlona o conmiserativa. Al no haber una salida, no era imaginable una entrada. Se consideraba, por otro lado, señal de gran cultura e información conformarse con el hecho de estar allí, sin buscar para ello un sentido o una razón. La conciencia de no caer en el autoengaño llenaba a los sabios de orgullo, por lo que se autotitulaban "desengañados" o "desengañadores". Por lo mismo, en todo el pueblo de sombras sólo tenía valor de verdadero lo que iba acompañado del amargo sabor del desengaño.

La prisión de la libertad

Michael Ende (2006: 112)

\section{Retos para las ciencias sociales y los nuevos paradigmas emergentes}

Como se pudo observar en la parte precedente de este trabajo, las ciencias sociales se formaron y consolidaron en Europa en el siglo XIX. Sus bases no han cambiado sustancialmente, aunque ya a mediados del siglo XX empezaron a aparecer cada vez más dudas y críticas al respecto, que hoy se están agudizando. Por lo tanto, resulta importante plantear algunas reflexiones en torno a la función e importancia del conocimiento y el conocer, para luego ver los retos que surgen, para el conocimiento y las ciencias sociales, desde algunos de los nuevos problemas y paradigmas emergentes, como el tema ambiental, así como de las exigencias de reflexión desde la perspectiva de la colonialidad.

Considerando los problemas que están enfrentando hoy -comienzos del siglo XXI- las sociedades del mundo, hay que preguntarse por la pertinencia de las bases modernas liberales del siglo XIX que se mantienen 
persistentemente en muchas áreas de las ciencias sociales. El abanico de problemas es casi sin fin y abarca las injusticias socioeconómicas cada vez más pronunciadas; la desintegración de muchas redes sociales, y, a la vez, la reestructuración de nuevos tipos de redes (por ejemplo virtuales); el desarraigo cultural por desplazamientos y migraciones masivos, ya sea por causas económicas, de violencia o ambientales; los cambios por la homogeneización de los patrones culturales en todo el mundo (comida, tipo de vestido, televisión, cine, etc.); la violencia, las guerras y los conflictos locales, regionales y globales; así como las sin precedentes transformaciones antrópicas masivas sobre el entorno natural, que han generado degradación, contaminación y destrucción, y cuyas consecuencias e implicaciones a largo plazo aún no son del todo previsibles en su conjunto.

Si se supone que una civilización es una organización social que ha logrado establecer, para un número de personas considerable, un orden socioeconómico, legal y cultural por medio de la superación de la "ley del más fuerte”, con lo cual intenta garantizar una convivencia pacífica, entonces se puede hablar actualmente de una crisis civilizatoria, ya que estas premisas están fallando, como evidencian los múltiples problemas ya mencionados. Además, si se está viviendo actualmente en un mundo en el que los especialistas (economistas, antropólogos, sociólogos, etc.) -formados en universidades y en la clásica división entre ciencias duras y ciencias sociales con sus respectivos cánones- son los que tienen cierta injerencia en las decisiones de mayor envergadura (sociales, políticas, económicas, ambientales), se plantea la siguiente pregunta: ¿se pueden solucionar, o al menos mitigar, algunos de los problemas complejos que aquejan a las sociedades, si se toman como base concepciones y supuestos deterministas, lineales, estáticos y/o fragmentados?

En este trabajo se parte de la hipótesis de que esta visión ha generado en gran medida estos problemas, y que no podrá ofrecer "soluciones" satisfactorias a largo plazo por estar atrapada dentro de sus propias visiones, que le impiden ver alternativas. Efectivamente, se observa que en los distintos ámbitos sociales y académicos, en muchos lugares y regiones del mundo, hay una gran búsqueda de caminos diferentes, porque se reconoce que las soluciones tradicionalmente ofrecidas (por Occidente) ya no son ni suficientes ni satisfactorias. Este hecho se refleja también en la gran diversidad de grupos sociales que se vienen reuniendo (por ejemplo, en el Foro Social Mundial), o en la bibliografía creciente sobre nuevos horizontes, o en la constatación de la existencia de los más diversos grupos académicos.

Tal vez uno de los aspectos que hace que esta crisis civilizatoria sea más evidente, y que está relacionado con las formas de conocer y sus formas destructivas de apropiación del entorno natural, es el problema ambiental, que es un elemento (factor, dimensión o aspecto) que el conjunto de las ciencias sociales no ha logrado tener en cuenta o, más bien, ha excluido de sus reflexiones y teorizaciones. Y es precisamente el problema ambiental el que ha puesto de manifiesto tanto la insustentabilidad de este modelo civilizatorio como su incapacidad de encontrar soluciones satis- 
factorias a largo plazo (ver también Leff 1998). Esta exclusión está relacionada con el hecho de que la dimensión ambiental trata de la vida -un sistema abierto, impredecible, cambiante, no lineal-, que difícilmente puede ser pensada desde sistemas cerrados como los que propone la modernidad.

Frente a este escenario, conviene señalar los grandes retos que tiene el ser humano moderno para readaptarse de manera más armónica a su entorno y, por ende, a sus semejantes (y viceversa). Esta idea de adaptación al medio a través del conocimiento se puede argumentar desde tres auto$\mathrm{res}^{3}$ : Humberto Maturana, Francisco Varela y Augusto Ángel Maya. Los dos primeros argumentan desde la biología y la neurociencia; Ángel Maya, desde la filosofía y la historia.

Para Maturana y Varela, los seres vivos tienen tres características fundamentales. Primero, son seres vivos al constituirse en unidades; segundo, tienen una identidad autopoiética con una red histórica propia de linajes; finalmente, nacen como resultado del acoplamiento entre células descendientes de una sola (ver también Floriani 2004). La meta que se proponen los autores desde esta perspectiva es "entenderse a sí mismos como producto del mismo mecanismo que permite hacer la pregunta sobre cómo uno se puede reconocer en el mundo [y] llevar al reconocimiento de que disponemos solamente de un mundo" (Maturana, Varela 1987: 19). Desde este punto de partida, la pregunta guía que estos dos autores se plantean es: "¿qué sucede en un sistema viviente de tal manera que tenga la capacidad, en un área determinada -incluso con el lenguaje-, de operar de manera efectiva y exitosa?” (Schneider 1998: 198). Maturana y Varela no preguntan por el significado, la información o la verdad que pueda tener el conocimiento (las preocupaciones clásicas de la epistemología desde la filosofía), sino por los mecanismos y procesos internos del organismo que lo hacen posible. Como dicen, "la epistemología debe demostrar cómo el conocer genera la explicación del conocimiento [...] que es un punto de partida muy diferente al común, que parte del supuesto de que el fenómeno de la explicación y el fenómeno explicado pertenecen a áreas diferentes" (Maturana, Varela 1987: 257). De esta manera, los dos autores llegan a la definición del concepto conocer, como "un acto efectivo, es decir, efectividad operacional en el área existencial del ser vivo [...] el conocer humano como una actividad efectiva pertenece por lo tanto al área biológica pero siempre es vivida en una tradición cultural” (Maturana, Varela 1987: 35, 261). En ese sentido, conocer es un acto efectivo, que se vincula con respuestas adaptativas: "en cuanto conocemos cómo conocemos, nos creamos a nosotros mismos" (Maturana, Varela 1987: 262). Floriani sintetiza muy bien en qué consiste el "árbol del conocimiento" del que hablan Maturana y Varela es "una discusión sobre las formas y los mecanismos circulares (no necesariamente viciosos) en el que la mente (= sistema nervioso), la evolución de los individuos y de las especies y el medio ambiente se acoplan estructuralmente” (Floriani 2004: 84).

Para Maturana y Varela, la cultura es una parte estructural del conocimiento, un caso especial de la conducta comunicativa. El conocimiento 
es el resultado estructural de lo orgánico y lo ambiental. Por lo tanto, estos autores llaman la atención sobre la responsabilidad que supone el conocer, ya que, por ejemplo, las tradiciones cultural y biológica, que corresponden a las regularidades de un acoplamiento de un grupo social, no se pueden considerar solamente como formas de ver y actuar sino también como formas de ocultar. Esto significa que puesto que la tradición cultural está inmersa en aquellos tipos de comportamiento que se dan por supuestos y que aparentemente no requieren de reflexión alguna, solamente se genera reflexión cuando ya no funcionan ciertos comportamientos (Maturana, Varela 1987: 261). Además, el conocer obliga a adquirir una posición de constante alerta frente a la tentación de la certeza, ya que "el centro de todas las dificultades, con las cuales nos vemos confrontados hoy en día, es nuestro desconocimiento del conocimiento y nuestro no-saber del saber" (Maturana, Varela 1987: 268). Floriani sintetiza esta responsabilidad que se adquiere por el conocimiento del conocimiento, en cuanto observadores que manejamos los códigos de la ciencia, que nos obliga a:

a) No ceder a la tentación de la certeza

b) Entender que las certezas no son pruebas de verdad

c) Al saber que sabemos, no podemos negar que sabemos

d) Mirar hacia una situación desde una perspectiva más amplia y distante

e) Que el otro también tenga lugar en nuestro dominio de las experiencias y que podamos construir un mundo con él

f) Inclusión en el mundo, entre otras cosas, del fenómeno de las identidades personales

g) Darse cuenta de que, como humanos, sólo tenemos el mundo que creamos con los otros (Floriani 2004: 90).

Es de anotar que estas siete observaciones llevan implícito el principio de precaución, que se ha perdido en el conocimiento hegemónico moderno.

Ángel Maya (1996, 2000, 2002) parte desde otro ángulo argumentativo, para mostrar igualmente la adaptación al medio a través del conocimiento o, más bien, de la cosmovisión, argumentando que la cultura, y los instrumentos que ella construye, representan una forma específica de adaptación al medio. En este orden de ideas, cada cosmovisión determina primero qué posición e importancia se les asignan tanto al ser humano como a la naturaleza; es decir, determina si el ser humano hace parte intrínseca del entorno natural, si es determinado por las leyes naturales o si es un ser superior a la naturaleza y por ende puede dominarla explotarla o respetarla. La cosmovisión es construida por el propio ser humano, que es la única especie capaz de adaptarse a todos los ecosistemas del planeta (desde el Polo Norte hasta el Sahara, pasando por el Amazonas) y organizarse a través y alrededor de instrumentos y símbolos, con efectos muy concretos sobre el manejo y uso de los respectivos recursos de su entorno, lo cual no siempre se produce de manera evidente o consciente. Como señala muy elocuentemente Ángel Maya, el ser humano ha dejado de adaptarse al medio, en su evolución, de manera fisiológica, para seguir 
haciéndolo a través de una plataforma instrumental. Es decir, el animal humano, para poder sobrevivir, tiene que transformar su entorno natural, trastocando sus leyes ecológicas. Esto tiene como consecuencia el hecho de que

[e]s la primera vez que el equilibrio depende de responsabilidades. La ciencia y la tecnología han venido transformando los equilibrios anteriores. La historia no es más que un proceso de modificación y domesticación de la naturaleza en su conjunto. La cultura es naturaleza transformada y la naturaleza ha entrado en un proceso de hominización (Ángel Maya 1996: 52)

Lo anterior significa que la cultura resulta ser una estrategia adaptativa compleja, y el problema ambiental no podrá ser reducido a un simple ecologismo o conservacionismo de la naturaleza, sino que resulta preciso entender la estrecha interrelación de cultura-técnica y entorno natural, así como las implicaciones de los usos tecnológico y energético sobre los frágiles equilibrios ecosistémicos, que están mediados por mundos simbólicos. Ángel Maya entiende por mundo simbólico

la compleja estructura del lenguaje que codifica la experiencia social. Recoge las diferentes miradas culturales sobre el mundo. Sirve tanto para afianzar la experiencia social como para criticarla [...] el mundo simbólico recoge las pautas del quehacer social, organiza el mundo de los significados en un tejido filosófico, articula el lenguaje del conocimiento científico y expresa la sensibilidad poética o el relieve de la experiencia artística, al mismo tiempo que establece los preceptos éticos o jurídicos (Ángel Maya 1996: 87).

En este mundo simbólico entran por lo tanto la ética social, el derecho, la filosofía, la literatura, la poesía, la ciencia, el mito, la religión, y por ende también las ciencias sociales y humanas occidentales, como una forma muy particular de conocer y como generadoras de un conocimiento específico/limitado.

Si se comparan ahora las dos lecturas de adaptación al medio a través del conocimiento y de los instrumentos culturales, se puede concluir que ambas resultan indispensables para la supervivencia del ser humano y son naturales. Ante este panorama surgen algunas preguntas:

1. Si el conocimiento es una forma de adaptación al medio, ¿qué le está pasando hoy, que, en vez de adaptarse, pone en peligro la supervivencia de la vida en el planeta (transgénicos, armas, energía nuclear, etc.)?

2. Si la cosmovisión dominante se fundamenta hoy en la modernidad, y por ello en la ciencia y sus múltiples disciplinas, las ciencias sociales desempeñan un papel importante en este contexto. Entonces, ¿qué capacidad tienen aún estas ciencias sociales para adaptarse a un mundo constantemente cambiante, profundamente emergente, 
caótico e imprevisible, con un marco teórico decimonónico, mecanicista, lineal?; ¿se han convertido estas ciencias sociales en una jaula que no permite ver, explorar y experimentar otros caminos posibles?

Ciertamente se vienen observando en las últimas décadas discusiones fuertes alrededor de la vigencia, los problemas y los límites entre las diferentes ciencias y sobre las ciencias mismas. De Sousa Santos (2004) realiza una compilación bastante amplia y rica de esta discusión, que reúne en un libro con un título muy bello y acertado: Conocimiento prudente para una vida decente. En él se señala claramente que el debate entre las ciencias gira en torno a la naturaleza y a la validez del conocimiento científico que legitima y produce las grandes transformaciones (políticas, sociales, tecnológicas y ambientales), así como alrededor del sentido y la naturaleza de las transformaciones del mundo que ha propiciado el conocimiento, teniendo muy en claro que desde el siglo XVII se privilegia la epistemología moderna. En el fondo del debate hay diferentes posiciones con respecto a la concepción de ciencia y al papel que tiene ésta en la transformación política de la sociedad. De Sousa señala que el realismo y el positivismo científico entraron en crisis cuando aparecieron conceptos en las teorías como contingencia, incertidumbre, complejidad, irreversibilidad, etc. Pero también se vienen generando cambios en las ciencias sociales desde cuando aparecieron los estudios culturales, estudios feministas, sobre razón y etnia y estudios sobre la ciencia en sí, que terminan cuestionando profundamente la ciencia y la razón misma, aquella razón analítica, fragmentadora y reductora, que ha llegado a sus límites al verse confrontada con la complejidad del ser y el acontecer.

Los cuatro capítulos del libro de De Sousa muestran la variedad y riqueza que ha tenido la discusión, abriendo con ello un sinfín de posibles lecturas innovadoras y alternativas de, y a, los múltiples problemas que aquejan a las sociedades. Pero sobre todo, estos capítulos son un abanico relativamente representativo de aspectos centrales del pensamiento sobre las ciencias, que han generado discusiones y cambios; son temas y aspectos que ponen en evidencia sobre todo el problema de las ciencias fragmentadas. Allí se muestra cómo en las orientaciones reduccionistas se contraponen dos perspectivas: una cultura científica versus una cultura humanista. Esas orientaciones están cediendo su lugar a la complejidad, a la trans- y a la interdisciplinariedad. En esta discusión, se hace evidente que será necesario recuperar la filosofía que se divorció de la ciencia, precisamente para poder recuperar/lograr un conocimiento más humano y, agrego yo aquí, más ambiental.

Ligado a este problema de la fragmentación, De Sousa cuestiona también la línea divisoria entre las ciencias naturales y las sociales. Él propone tres ejes para superar esta división: a través del estilo cognitivo de la mecánica cuántica, de la lingüística, y de los autómatas celulares y programas computacionales.

Pero también se cristaliza otro tema fundamental, el de la defensa de la autonomía del conocimiento; se debe llamar la atención sobre el he- 
cho de que si antes la defensa era respecto a los poderes políticos y a las ideologías totalitarias, hoy ella tiene que darse ante la mercantilización de la ciencia, y la conversión de la ciencia y la tecnociencia en entes comerciales. Es decir, es necesario reconocer el poder que tienen, por ejemplo, por su capacidad financiera, las grandes trasnacionales farmacéuticas en las investigaciones médicas, o la influencia de las consultorías en las facultades de ciencias sociales respecto a sus objetos de investigación ${ }^{4}$. Esto también va estrechamente ligado con la problematización de la relación entre ciencia y valores, y resulta indispensable distinguir entre imparcialidad, autonomía y neutralidad de la ciencia, en el momento de determinar prioridades y orientaciones de investigación, en la evaluación y en la aplicación misma de la ciencia. De Sousa lo sintetiza muy bien diciendo:

Sobre la forma del Consenso de Washington, tal modelo [capitalismo neoliberal] pasó a constituir el núcleo duro de un proyecto político global que consistió (y consiste) en someter a las leyes y a la lógica del mercado a un conjunto cada vez más amplio de interacciones, de modo que se pasa aceleradamente, y a nivel global, de la economía de mercado hacia la sociedad de mercado (De Sousa Santos 2004: 48) .

Es éste el punto en que las reflexiones sobre la ciencia deben evidenciar y aclarar el papel que desempeña la "neutralidad" científica; asumir la responsabilidad que confiere el conocimiento; mostrar los límites de la validez del conocimiento científico; e iniciar un diálogo, sin jerarquías, con otros conocimientos, todo esto con el fin de evitar que el conocimiento sea cooptado por unos pocos en beneficio de otros pocos.

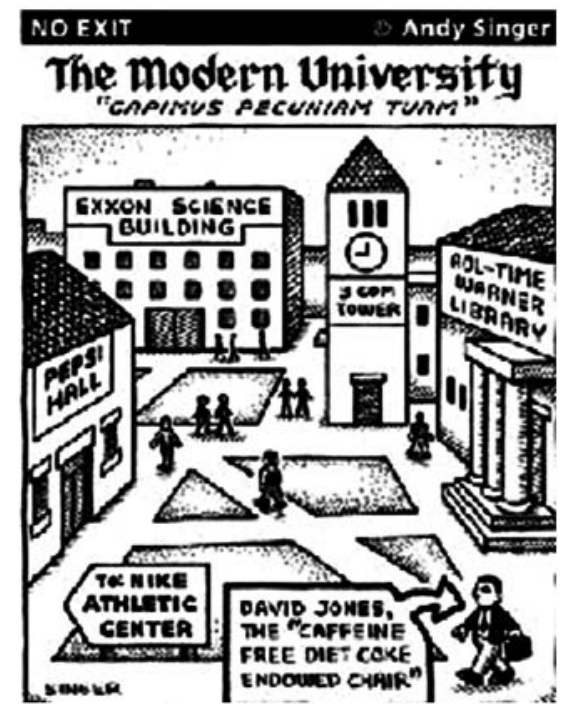

http://firgoa.usc.es/drupal/node/22778 Consultado: 2007 
Esta caricatura recoge muy bien el problema que se está presentando cada vez con más fuerza en la educación, en la cual Inglaterra es uno de los pioneros, con sus estrategias de "Public-Private Partnerships for Schools"6.

Pero las ciencias no podrán realizar, por sí solas, estas labores: tendrán que acudir a la filosofía, ya que, como bien lo señala Heidegger, al igualar la cognición con la tecnología y el cálculo, la ciencia ya no piensa. Este autor lo expresa muy bellamente al decir que

cuando planificamos, investigamos, organizamos una empresa, contamos ya siempre con circunstancias dadas. Las tomamos en cuenta con la calculada intención de unas finalidades determinadas. Contamos de antemano con determinados resultados. Este cálculo caracteriza a todo pensar planificador e investigador. Semejante pensar sigue siendo cálculo aun cuando no opere con números ni ponga en movimiento máquinas de sumar ni calculadoras electrónicas. El pensamiento que cuenta, calcula; calcula posibilidades continuamente nuevas, con perspectivas cada vez más ricas y a la vez más económicas. El pensamiento calculador corre de una suerte a la siguiente, sin detenerse nunca ni pararse a meditar. El pensar calculador no es un pensar meditativo: no es un pensar que piense en pos del sentido que impera en todo cuanto es. Hay así dos tipos de pensar, cada uno de los cuales es, a su vez y a su manera, justificado y necesario: el pensar calculador y la reflexión meditativa (Heidegger 1994: 18).

En un mundo que claramente se ha dejado llevar por el pensar calculador, lo cual se refleja especialmente en la racionalidad instrumental, se requiere urgentemente de una reflexión meditativa que dé cuenta de las formas de operar, actuar y justificar de la ciencia, en este caso de las ciencias sociales, del sentido y de las implicaciones del conocimiento sobre la sociedad y, principalmente, sobre el entorno natural.

Los nuevos paradigmas emergentes en este contexto tienen un papel crucial, que en este artículo apenas se van a mencionar; profundizar en ellos sería objeto de otro texto. En primera instancia resulta importante señalar la influencia e impacto que han tenido tanto las ciencias de la complejidad como los estudios culturales sobre las ciencias clásicas (tanto naturales como sociales/humanas) (ver Wallerstein 2005). A la vez, desde las ciencias de la complejidad han surgido conceptos como indeterminación, incertidumbre, riesgo, resiliencia, emergencia, autoorganización, autosuficiencia, irreversibilidad, entropía, etc.; cada uno de estos conceptos cuestiona profundamente la ciencia normal newtoniana, la cual había sido retomada y aplicada en los más diversos ámbitos, y fraguado profundamente casi todo el pensamiento. Por lo tanto, ¿qué significa aplicar la entropía a las teorías económicas? Georgescu-Roegen, padre de la economía ecológica, ya lo ha demostrado desde los años 1970. ¿Qué implicaciones tienen conceptos como riesgo y resiliencia para pensar en grupos sociales menos favorecidos?, ¿qué opciones ofrecen los conceptos de emergencia y 
autoorganización para pensar en movimientos sociales?, ¿qué implica considerar la entropía y la irreversibilidad (ambiental) en la toma de decisiones políticas energéticas?

Por su parte, los estudios culturales critican el tipo de conocimiento de las humanidades, institucionalizadas como dogma opuesto a la ciencia (Wallerstein 2005). A estos estudios culturales se dedicaron especialmente los literarios, los antropológicos y las nuevas cuasidisciplinas, al unir tres temas:

Primero, la importancia central, para el estudio de los sistemas sociales históricos, de los estudios de género y todos los tipos de estudios "no eurocéntricos"; segundo, la importancia del análisis histórico local, muy ubicado, que muchos asocian con una nueva "actitud hermenéutica”; tercero, la estimación de los valores asociados con las realizaciones tecnológicas y su relación con otros valores (Wallerstein 2001: 71).

Esta combinación ha logrado desafiar las teorías existentes de las ciencias sociales y está desempeñando un papel importante en la legitimación y la producción de conocimiento.

En una línea similar, pero más latinoamericana, se pueden plantear los aportes de la modernidad/colonialidad, que ponen en tela de juicio a las ciencias sociales por ser hegemónicas y eurocéntricas en su concepción de modernidad $^{7}$. Con conceptos como colonialidad del poder, que plantea al capital como un poder hegemónico que ha articulado raza, trabajo, espacio y género según sus necesidades (ver trabajos de A. Quijano); o del eurocentrismo, que estos estudios entienden como un modelo de conocimiento europeo, que se presenta como el parámetro central, universal, verdadero y objetivo, y por ende hegemónico; ese es el llamado punto cero del que habla Castro-Gómez, un parámetro que parte y se fundamenta en la experiencia histórica local europea, un eurocentrismo que posibilita la colonialidad del saber (ver trabajos de Lander y Castro-Gómez). Desde estas conceptualizaciones de la modernidad/colonialidad ya no se podrán ver como "universalmente válidos”, para el análisis social, conceptos como Estado-nación, clase social, mercado, capital, poder, y muchos otros clásicos de las ciencias sociales.

Y especialmente en torno a la problemática ambiental se requiere de un pensamiento reflexivo meditativo, que permita entender las implicaciones a largo plazo, las interdependencias e interrelaciones, la necesidad del principio de precaución y responsabilidad, puesto que el pensar calculador no ha logrado comprender esta complejidad. La diferencia de pensar los problemas ambientales desde estos ángulos significa abordarlos y entenderlos de manera no fragmentada, no lineal, y por ende las propuestas de solución también serán diferentes de las positivistas modernas.

En la forma tradicional de abordar un problema (por ejemplo, el derrame petrolero en el Golfo de México), se mostraron las siguientes "so- 
luciones” técnicas: para el control de la mancha de petróleo se realizó la dispersión de Coerxit (sin conocer su toxicidad en grandes volúmenes ni en espacios abiertos); se instalaron vallas de contención en las playas (que fueron arrancadas por las primeras olas fuertes); se hizo lavado de pelícanos a mano (se demostró que el $90 \%$ de estos animales no sobrevive al estrés del proceso); se dio una recompensa monetaria a la industria pesquera (¿se puede dar una recompensa por la pérdida de la cultura pesquera por sustracción indefinida de "materia” a las familias?); se dijo que habría exigencia de controles más severos para futuras perforaciones (nueva organización administrativa, sin cuestionamiento per se del sentido y de los niveles de consumo del petróleo; sin embargo, por la misma época permitieron la exploración petrolera en Alaska, parque natural por excelencia). En resumen, se trata de "soluciones" puntuales, instrumentalistas, el resultado de análisis y perspectivas positivistas, que, a largo plazo, no garantizan la supervivencia de la vida.

En cambio, si se aborda este mismo problema ambiental del derrame de petróleo desde una perspectiva alterna, se plantea primero otro tipo de preguntas: ¿cómo se lograron los permisos de exploración a semejantes profundidades?; ¿quiénes permitieron las alianzas "colegiales” entre los petroleros y las entidades estatales supervisoras?; ¿quiénes “necesitan” y para qué tanto petróleo?; ¿a qué tipo de necesidades responden las solicitudes?; ¿con qué racionalidad se justifican y legitiman estas perforaciones de alto riesgo ambiental?; ¿ alguien consideró el principio de precaución y el concepto de irreversibilidad en la toma de decisiones?; ¿en qué términos de tiempo se piensa (biológico, geológico, social) al adoptar decisiones?; ¿los daños ambientales son compensables con valores crematísticos?; en caso de que lo sean, ¿quiénes y cómo se fijan los “valores” monetarios, en términos entrópicos? ¿este tipo de perforaciones es sustentable a largo plazo?; ¿qué noción de riesgo se maneja en estas empresas petroleras?; ¿cómo hacer entender que todos somos responsables de este desastre puesto que somos los consumidores de los infinitos derivados de los productos petroleros?; ¿cómo abordar y pensar este problema desde conceptos como irreversibilidad, entropía y resiliencia?; ¿qué idea de desarrollo subyace a, y justifica, estas actividades petroleras?

Otro aspecto evidenciado nuevamente por esta catástrofe ambiental es que los supuestos avances tecnológicos de punta no son capaces de dominar la naturaleza imprevisible, y menos cuando se está en conjugación con los errores humanos, que jamás se podrán eliminar (como ya lo demostró también Chernóbil). Es decir, las pretensiones del conocimiento moderno de medir, calcular, predecir, dominar y explotar de forma infalible y perfecta no son reales y especialmente si se ven en combinación con la condición humana, la avaricia (¿qué teorías económicas la favorecen?) y el poder (¿qué teorías de las ciencias políticas y sociológicas lo justifican/ fomentan?).

Con este panorama, se plantea también la necesidad de un pensamiento ambiental que sea capaz de proponer una racionalidad ambiental y 
un saber ambiental (ver por ejemplo toda la obra de Leff), que supere las limitaciones epistemológicas del conocimiento moderno, para comprender la complejidad de los problemas ambientales; un pensamiento ambiental que posibilite pensar desde y para la vida, y no desde la perspectiva del objeto inerte, manipulable, sustituible.

\section{A manera de conclusión}

Llegados a este punto, más que conclusiones, se quieren plantear algunas consideraciones que se deberían tener en cuenta en el momento de trabajar con las ciencias sociales, ya sea aplicándolas, estudiando, enseñando o investigando. A partir de lo presentado hasta aquí, efectivamente se puede decir que las ciencias sociales se encuentran, en buena medida, enjauladas en viejas suposiciones, marcos y construcciones teóricas, y que aún son, además, una herramienta importante del pensamiento eurocéntrico hegemónico. Lentamente, como siempre ocurre en lo que respecta a procesos de cambio profundos, se va tomando conciencia de este problema en América Latina, y esto se refleja tanto en los estudios que se vienen realizando sobre modernidad/colonialidad como en los estudios culturales pero también desde el pensamiento ambiental. Resulta igualmente importante hacer énfasis en la relación directa que existe entre las teorías socioeconómicas/filosóficas y la construcción de realidades, pues las opciones epistemológicas determinan oportunidades de vida, como bien lo señalara De Sousa en el ya nombrado título de su libro: lo que se requiere es un conocimiento prudente para una vida decente.

Evidenciar los valores e intereses que subyacen a la ciencia (que no son neutrales); cuestionar las bases epistemológicas eurocéntricas de las ciencias sociales; recuperar la filosofía para entender el porqué y el para qué del conocimiento; establecer diálogos sin jerarquías entre los diferentes conocimientos y saberes (lo cual implica a la vez superar las fronteras disciplinarias); reconocer, entender y asimilar los avances, por ejemplo, de la biología evolutiva, con sus aportes en las ciencias de la complejidad, que actualmente es la que marca las pautas del conocimiento de punta, como anteriormente lo hiciera la física newtoniana). Éstos resultan ser los grandes retos, si se quieren abordar temas/problemas como el socioambiental, ya que está demostrado ampliamente que los modelos tradicionales no logran ofrecer ni explicaciones ni soluciones satisfactorias a los problemas socioambientales complejos. Sobre todo deberán plantearse preguntas como ¿qué significa y qué aporta pensar desde un enfoque ambiental?, ¿qué tan compatible es este enfoque con las teorías y los conceptos tradicionales de las ciencias sociales? o ¿qué sociedad y calidad de vida queremos a largo plazo? 


\section{Notas}

${ }^{1}$ Una buena exposición de este problema está en el trabajo de J. Mejía Navarrete, quien demuestra esta aceptación y reproducción del conocimiento eurocentrista, casi incondicional, rastreando los inicios de la sociología en el Perú: "[L]a sociología asume un carácter eurocéntrico: lo moderno en el Perú es sinónimo de lo extranjero, el predominio de la innovación y novedad del conocimiento provienen inicialmente de la producción bibliográfica europea, a partir de la cual se define una visión que considera el desarrollo en forma lineal, siguiendo un orden de etapas ascendentes según el modelo occidental”. Mejía; http:// esmiperu.blogspot.com/2007/10/la-sociologa-en-el-per.html Consultado 12.05.2005.

\section{${ }^{2}$ Ver también: Walsh, http://www.oei.es/salactsi/walsh.htm Consultado 07.09.2007. \\ ${ }^{3}$ La argumentación de estos tres autores la trabajé también en mi libro (2009). \\ ${ }^{4}$ Ver también el trabajo de Lander, Edgardo, La ciencia neoliberal, http://www.tni.org/ archives/lander/ciencianeoliberal.pdf, (consultado febrero 22 de 2007)}

${ }^{5}$ Resulta preciso ampliar la cita: "Subyacentes a tal proyecto están los siguientes principios: los valores que cuentan son reductibles a precios; todas las prestaciones de servicios son privatizables; la competición tiene prioridad total sobre la cooperación en todas las interacciones sociales: las condiciones de competitividad determinan el lugar que puede ser socialmente concedido a las condiciones de otros criterios de sociabilidad; la previsibilidad de los nexos causales entre acciones y sus consecuencias y el factor principal de estabilidad, independientemente de la sustancia de unas y otras; la innovación y la creatividad -dos condiciones esenciales de la competitividad- exigen la fragmentación de las competencias; la individualización de los contratos de trabajo (flexibilización para empresarios, precarización para los trabajadores); la prioridad total del corto plazo en los planes de vida de los productores; la innovación y la creatividad están sujetas al constante aval de los resultados y éste está dominado por criterios de rentabilidad; el mercado es la mejor (o única) garantía de eficiencia y de previsibilidad, innovación, creatividad, fragmentación y rentabilidad” (De Sousa Santos 2004: 48) (traducción libre de la autora).

${ }^{6}$ Ver por ejemplo (todos consultados el 27 de noviembre de 2007): http://www.idra.org/ IDRA_Newsletters/April_1999/Coca-Cola_Valued_Youth_Program: _The_Value_of_Video_Conferencing/ , http://www.edfacilities.org/rl/funding_partnerships .cfm , http://www.mackinac.org/article.aspx?ID=7511

${ }^{7}$ La modernidad/colonialidad parte de las siguientes premisas:

1. Un énfasis en localizar los orígenes de la modernidad en la conquista de América y el control del Atlántico después de 1492, antes que en la Ilustración o el final del siglo XVIII como es comúnmente aceptado. Una atención persistente al colonialismo y al desarrollo del sistema mundial capitalista como constitutivos de la modernidad; esto incluye la determinación de no pasar por alto la economía y sus concomitantes formas de explotación.

2. En consecuencia, la adopción de una perspectiva planetaria en la explicación de la modernidad, en lugar de una visión de la modernidad como un fenómeno intra-europeo.

3. La identificación de la dominación de otros afuera del centro europeo como una necesaria dimensión de la modernidad, con la concomitante subalternización del conocimiento y las culturas de esos grupos.

4. La concepción del eurocentrismo como la forma de conocimiento de la modernidad/ colonialidad -una representación hegemónica y un modo de conocimiento que arguye su 
propia universalidad- y que descansa en "una confusión entre una universalidad abstracta y el mundo concreto derivado de la posición europea como centro” (Dussel y Quijano, en: Escobar 2005: 70,71). 


\section{Bibliografía}

Ángel Maya, A. (2000), El retorno de Ícaro, la razón de la vida, muerte y vida de la filosofía, una propuesta ambiental. Pnuma, UNDP, IDEA, Bogotá.

Ídem (2000), La aventura de los símbolos. Una visión ambiental de la historia del pensamiento, Ecofondo, Bogotá.

Ángel Maya, A. (1996), El reto de la vida. Ecosistema y cultura, Ecofondo, Bogotá.

Bacon, F. (1984), Novum organum, Sarpe, Madrid.

Berman, M. (2001 [1981]), El reencantamiento del mundo, Cuatro Vientos, Chile.

Castro-Gómez, S. (2000), “Ciencias sociales, violencia epistémica y el problema de la 'invención del otro' ”, en: Lander, E., La colonialidad del saber: Eurocentrismo y ciencias sociales, Clacso, Buenos Aires. Disponible a texto completo en: http://www.clacso.org/wwwclacso/espanol/html/libros/ lander/7.pdf (consultado el 7 de septiembre de 2007).

Ídem (2005), La hybris del punto cero. Ciencia, raza e Ilustración en la Nueva Granada (1750-1816), Pontificia Universidad Javeriana, Bogotá.

Comte, A. (1996), Discurso sobre el espíritu positivo, Altaya, España.

De Sousa Santos, Boaventura (org.) (2004 [2003]), Conhecimento prudente para uma vida decente. Um discurso sobre as ciencias revistado, São Paulo, Ed. Cortez, Brasil.

Díaz, Á. (1991), “Los orígenes de la problemática curricular”, en: de Alba, A., Díaz, Á; González, É, El campo del currículum, Antología, Vol. I, CESU, México.

Elmusa, S. (ed.) (2005), "Culture and the Natural Environment. Ancient and Modern Middle Eastern Texts", Cairo Papers in Social Science, vol. 26, 1. American University in Cairo Press, Cairo.

Ende, M. (2006 [1997]), La prisión de la libertad, Alfaguara, México.

Eschenhagen, M. (2005), La educación ambiental superior en América Latina: ¿Cómo se refleja la interdisciplinariedad en los planes curriculares de maestría? Tesis doctoral para obtener el grado de Doctora en Estudios Latinoamericanos (Director, Dr. Enrique Leff), UNAM, México.

Ídem (2007), “¿Será necesario ambientalizar la educación ambiental?”, en: Noguera de Echeverri, Ana Patricia (comp.) Hojas de sol en la Victoria 
Regia, Emergencias de un pensamiento ambiental alternativo en América Latina, Ed. Universidad Nacional de Colombia Sede Manizales, Manizales.

Escobar, A. (1996), La invención del Tercer Mundo, construcción y deconstrucción del desarrollo, Norma, Bogotá.

Ídem (2005). Más allá del tercer mundo, globalización y diferencia. Icanh, Universidad del Cauca, Bogotá.

Floriani, D. (2004), Conhecimento, meio ambiente \& globalização, Ed. Juruá, PNUMA, Curitiba.

Giroux, H. (2003) (1983), Teoría y resistencia en educación, Siglo XXI y UNAM, México.

Ídem (1991), “Introducción y perspectivas del campo curricular”, en: de Alba, A., Díaz, Á.; González, É. El campo del currículum, Antología, Vol. I, CESU, México.

Grosfoguel, R. (2006), “La descolonización de la economía política y los estudios postcoloniales: transmodernidad, pensamiento fronterizo y colonialidad global”, en: Revista Tabula Rasa, Universidad Colegio Mayor de Cundinamarca, 4, 29, enero-junio 2006, Disponible a texto completo en: http://www.afyl.org/descolonizacioneconomia.pdf (consultado el 7 de septiembre de 2007).

Heidegger, M. (1994 [1959]), Serenidad, ODOS, Barcelona.

Lander, E. (comp.) (2000), La colonialidad del saber: Eurocentrismo y ciencias sociales, Perspectivas latinoamericanas, Unesco, Clacso, Argentina.

Lander, E. (1998), La ciencia neoliberal, Disponible a texto completo en: http://www.tni.org/archives/lander/ciencianeoliberal.pdf Consultado el 22 de febrero de 2007.

Leff, E. (1998), Saber ambiental: sustentabilidad, racionalidad, complejidad, poder, Siglo XXI, Pnuma, Ciich, México .

López Segrera, F. (2000), Abrir, impensar y redimensionar las ciencias sociales en América Latina y el Caribe. ¿Es posible una ciencia social no eurocéntrica en nuestra región?, Disponib er Baum der Erkenntnis, die biologischen Wurzeln menschlichen Erkennens, Scherz Verlag, München.

Mejía Navarrete, J. (2005), “La sociología en el Perú. Trayectoria histórica y desafíos teóricos”,Revista Investigaciones sociales Año IX No 15, Lima: Universidad Nacional Mayor de San Mrcos, pp. 251-275. Disponible a texto completo en: http://esmiperu.blogspot.com/2007/10/lasociologa-en-el-per.html $o$ http://sisbib.unmsm.edu.pe/bibvirtualdata/publi- 
caciones/inv_sociales/N15_2005/a12.pdf Consultado el 17 de septiembre de 2007.

Rostow, W. (1963) (1959), Las etapas del crecimiento económico, Fondo de Cultura Económica, México.

Schneider, N. (1998), Erkenntnistheorie im 20 Jahrhundert, klassische Positionen, Reclam Verlag, Stuttgart.

Spencer, H. (1905), Los primeros principios, Fernando Fe, Madrid.

Wallerstein, I.(2005 [2004]), Las incertidumbres del saber, Ed. Gedisa, Barcelona.

Wallerstein, I. (coord.) (2001 [1996]), Abrir las ciencias sociales, Siglo XXI, México.

Walsh, C. (2004), “Geopolíticas del conocimiento, interculturalidad y descolonialización”, Boletín ICCI-ARY Rimay, Año 6, No. 60, marzo de 2004, Disponible a texto completo en: http://icci.nativeweb.org/boletin/ 60/walsh.html Consultado el 7 de septiembre de 2007.

Ídem (2007), Las geopolíticas del conocimiento y colonialidad del poder. Entrevista a Walter Mignolo, Disponible a texto completo en: http:// www.oei.es/salactsi/walsh.htm Consultado el 7 de septiembre de 2007. http://firgoa.usc.es/drupal/node/22778 Consultado el 27 de noviembre de 2007.

http://www.idra.org/IDRA_Newsletters/April_1999/CocaCola_Valued_Youth_Program:_The_Value_of_Video_Conferencing/Consultado el 27 de noviembre de 2007.

http://www.edfacilities.org/rl/funding_partnerships.cfm Consultado el 27 de noviembre de 2007.

http://www.mackinac.org/article.aspx?ID=7511 Consultado el 27 de noviembre de 2007.

Recibido: 06.09.2010

Aceptado: 30.03.2011 